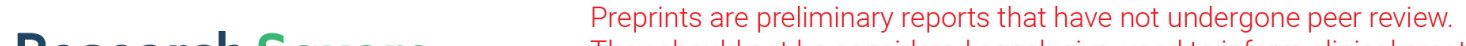 They should not be considered conclusive, used to inform clinical practice, or referenced by the media as validated information. \\ Risk Factors of Opioid use Associated with an Enhanced-Recovery Programme after Total Knee Arthroplasty
}

\section{Guorui Cao}

Luoyang Orthopaedic Hospital of Henan Province

Fuxing Pei ( $\Delta$ peifuxingwestchina@163.com )

Sichuan University West China Hospital https://orcid.org/0000-0003-3736-4724

\section{Research article}

Keywords: Opioid, Risk factors, Total knee arthroplasty, Length of stay

Posted Date: February 16th, 2021

DOI: https://doi.org/10.21203/rs.3.rs-241590/v1

License: (c) (1) This work is licensed under a Creative Commons Attribution 4.0 International License.

Read Full License

Version of Record: A version of this preprint was published at BMC Musculoskeletal Disorders on December 1st, 2021. See the published version at https://doi.org/10.1186/s12891-021-04937-8. 


\section{Abstract}

Background: The relationship between total knee arthroplasty (TKA) and postoperative opioid use is poorly studied. The aim of the study is to examine the prevalence and risk factors for opioid use with an enhanced-recovery programme after primary TKA.

Methods: We identified 361 patients undergoing TKA, and separated those on the basis of whether to receive opioid use after surgery. Multivariate logistic regression model was used to identify independent risk factors for opioid use after primary TKA. Length of stay (LOS) and postoperative complications were recorded and compared.

Results: The prevalence of opioid use after primary TKA was $23.0 \%$. Significant risk factor were more operative time $(\mathrm{OR}$ [odds ratio] $=1.017,95 \% \mathrm{Cl}$ [confidence interval] $=1.001$ to $1.032, p=0.034$ ) and protective factor was tranexamic acid use $(\mathrm{OR}=0.355,95 \% \mathrm{Cl}=0.161$ to $0.780, p=0.010)$. In addition, the LOS was longer in opioid group $(p<0.05)$.

Conclusion: Considering the adverse health effects of opioid use, strategies need to be developed to prevent persistent opioid use. Reducing operative time and application of tranexamic acid could lower the risk of opioid use with an enhanced-recovery programme after primary TKA.

\section{Introduction}

Total knee arthroplasty (TKA) is a common procedure to treat advanced osteoarthritis of the knee and the number of TKAs performed is growing annually $[1,2]$. Significant pain in the early postoperative period following TKA is common and effective pain control continued to be a challenging issue $[3,4]$. Appropriate pain management consisted of patient education, physical therapy and medications, including acetaminophen, non-steroidal anti-inflammatory drugs (NSAIDs) and opioid [5].

Opioid use is an effective method to treat choric pain after TKA, however, it is reported that postoperative opioid utilization have only a small clinical benefit but a high risk of side effects, such as delayed rehabilitation, higher revision risk, opioid dependency and withdrawal [6-8]. Considering the bad effects of opioid use, NSAIDs become important alternatives for postoperative pain care with the development of enhanced-recovery programme. Majority of patients could gain the optimization of pain control with intervention of NSAIDs, but opioid use was still a remedial measure for a few patients after primary TKA [4].

The risk factors for prolonged opioid use and opioid-related adverse drug events after TKA were extensively studied in previous studies [9-12]. However, the prevalence and related factors associated with whether or not use opioid after primary TKA was still poorly studied. Therefore, we perform this retrospective cohort study to investigate the risk factors associated with postoperative opioid use following primary TKA. 


\section{Materials And Methods}

\section{Study design and study sample}

We conducted a single-center retrospective cohort study of patients undergoing primary TKA owing to osteoarthritis and rheumatoid arthritis from June 2016 to January 2019. The study was approved by the hospital's Institutional Review Board. The patients were exclude with any of the following situations: cardiovascular problems, history of chronic musculoskeletal pain or other types of chronic pain, history of cancer and incomplete medical records.

Overall, 361 patients were included in this study. The enrolled patients were divided into opioid group or non-opioid groups according to whether patients received opioid drugs to control pain after primary TKA.

\section{Surgical Technique and Perioperative protocol}

All the surgeries were performed under general anesthesia by the same senior surgeon, through a midline skin incision, medial parapatellar approach, and a measured resection technique. The prosthesis was cemented posterior-stabilized prosthetic design with patellar resurfacing. $200 \mathrm{mg}$ ropivacaine diluted in $100 \mathrm{~mL}$ of normal saline solution was injected periarticularly when the deep fascia was sutured. No tourniquet, nerve block, intravenous patient-controlled analgesia or blood salvage system were used. The operative time and intraoperative blood loss were recorded carefully.

All patients received enhanced recovery protocols, including blood management, sleep management and so on $[13,14]$. The physical prophylaxis and chemoprophylaxis were applied to prevent DVT and PE on the basis of our previous study [3]. The TXA $(20 \mathrm{mg} / \mathrm{kg})$ was administered intravenously 5-10 minutes before skin incision, along with $1 \mathrm{~g}$ intravenous TXA (diluted in $100 \mathrm{~mL}$ of normal saline solution) at 3,6 , 12 , and 24 hours postoperatively.

Routinely, all patients received oral Celecoxib (200 mg twice a day, Celebrex; Pfizer, New

York) preoperatively and oral enteric-coated diclofenac sodium (50 mg twice a day, Voltaren; Novartis, Basel, Switzerland) postoperatively for 2-4 weeks after surgery. Cold pack and intermittent foot slope pump systems were given to all patients for 12-24 hours postoperatively. The opioid (OxyContin or oxycodone hydrochloride sustained release tablets, $10 \mathrm{mg}$ twice a day) was applied when the Visual Analogue Scale (VAS) score in motion was $\geq 5$ or $\geq 3$ in rest.

\section{Outcome Measurements}

The number of patients receiving opioid prescriptions after TKA was the outcome of interest. Perioperative variables considered as potential risk factors, including age, gender, diagnosis, body mass index, medical comorbidities and laboratory markers were collected. In addition, American Society of Anesthesiologists physical status classification, the application of TXA and drainage, operative time, 
intraoperative blood loss, length of stay (LOS) after surgery and postoperative complications were carefully recorded.

\section{Statistical analyses}

Descriptive and univariate analyses were conducted to compare the characteristics in patients with or without opioid use after primary TKA. A multivariable logistic regression model was used to examine independent risk factor for postoperative opioid use in TKA. Factors associated with postoperative opioid use with $p<0.10$ were selected for inclusion in the multivariable model. Collinearity was checked using tolerance values $(p>0.10)$. All analyses were performed using SPSS version 22.0(SPSS Inc. USA). A $p$ value of $<0.05$ was considered statistical significant.

\section{Results}

Totally, 361 patients were enrolled in the study. Among them, 83 patients received opioid drugs to treat pain after primary TKA. The prevalence of opioid use was $23.0 \%$. The results of comparison between opioid and non-opioid patients were shown in Table 1. The variables that were selected for logistic regression model after univariate analyses were smoking, TXA use, more operative time and intraoperative blood loss $(p<0.10)$. 
Table 1

The comparison of patients with or without opioid use following primary TKA

\section{Baseline Characteristic}

Demographic characteristics

$$
\operatorname{Age}(\bar{X} \pm S)
$$

Sex (male/female)

$\operatorname{BMI}\left(\bar{X} \pm S, \mathrm{~kg} / \mathrm{m}^{2}\right)$

Diagnosis (RA/OA)

$5 / 78$

$48.7 \pm 8.9$

$4.9 \pm 0.7$

$45(54.2 \%)$

$8(9.6 \%)$

Preoperative valgus $(\mathrm{N}, \%)$

\section{Comorbidities ( $\mathrm{N}, \%)$}

Smoking

Alcohol

Hypertension

Diabetes

Coronary heart disease

Preoperative anemia

COPD

\section{Preoperative laboratories}

$\mathrm{HB}(\mathrm{g} / \mathrm{L})$

Hct

Albumin $(\mathrm{g} / \mathrm{L})$

$66.1 \pm 8.9$

$19 / 64$

$25.6 \pm 3.5$

$10(12.0 \%)$

$5(6.0 \%)$

$10(12.0 \%)$

$10(12.0 \%)$

$8(9.6 \%)$

$39.7 \pm 3.7$

$42.8 \pm 3.2$
$17(6.1 \%)$

$16(5.8 \%)$

0.927

48 (57.8\%)

$19(22.9 \%)$

$131.3 \pm 13.4$

BMI: body mass index; RA: rheumatoid arthritis; OA: osteoarthritis; HSS: hospital for special surgery knee score; VAS: visual analogue scale; COPD: chronic obstructive pulmonary disease; HB:

hemoglobin; Hct: hematocrit; ESR: erythrocyte sedimentation rate; CRP: C reaction protein; IL-6: interleukin-6; PT: prothrombin time; APTT: activated partial thrombin time; ASA, American Society of Anesthesiologists; TXA: Tranexamic acid.

$p$ value calculated using independent t-test, Pearson chi-square test or Fisher exact test.

*Significant difference 


\begin{tabular}{|c|c|c|c|}
\hline Baseline Characteristic & Opioid group, $\mathrm{N}=83$ & Non-opioid group, $N=278$ & $p$ \\
\hline $\operatorname{ESR}(\mathrm{mm} / \mathrm{h})$ & $24.0 \pm 16.3$ & $27.0 \pm 18.0$ & 0.259 \\
\hline $\mathrm{CRP}(\mathrm{mg} / \mathrm{L})$ & $3.5 \pm 4.28$ & $3.6 \pm 3.0$ & 0.748 \\
\hline IL-6 (mg/L) & $4.3 \pm 2.9$ & $4.2 \pm 4.1$ & 0.771 \\
\hline Platelet $\left({ }^{\star} 10^{9} / \mathrm{L}\right)$ & $183.5 \pm 68.6$ & $187.6 \pm 59.6$ & 0.635 \\
\hline PT (sec) & $11.4 \pm 0.7$ & $11.3 \pm 1.0$ & 0.670 \\
\hline APTT (sec) & $27.7 \pm 3.1$ & $27.5 \pm 3.3$ & 0.751 \\
\hline \multicolumn{4}{|l|}{ Operative variables } \\
\hline ASA class $(\mathrm{N}, \%)$ & & & 0.789 \\
\hline $1-2$ & $70(84.3 \%)$ & $231(83.1 \%)$ & \\
\hline$\geq 3$ & $13(15.7 \%)$ & $47(16.9 \%)$ & \\
\hline Drainage use $(\mathrm{N}, \%)$ & $22(26.5 \%)$ & $88(31.7 \%)$ & 0.371 \\
\hline TXA use $(\mathrm{N}, \%)$ & $71(85.5 \%)$ & $259(93.2 \%)$ & $0.030 \star$ \\
\hline Dexamethasone use (N, \%) & $65(78.3 \%)$ & $234(84.2 \%)$ & 0.214 \\
\hline Operative time $(\mathrm{min})$ & $80.4 \pm 16.2$ & $76.7 \pm 15.0$ & 0.056 \\
\hline Intraoperative blood loss (mL) & $127.4 \pm 33.0$ & $104.6 \pm 29.2$ & $<0.001 *$ \\
\hline \multicolumn{4}{|c|}{$\begin{array}{l}\text { BMI: body mass index; RA: rheumatoid arthritis; OA: osteoarthritis; HSS: hospital for special surgery } \\
\text { knee score; VAS: visual analogue scale; COPD: chronic obstructive pulmonary disease; HB: } \\
\text { hemoglobin; Hct: hematocrit; ESR: erythrocyte sedimentation rate; CRP: C reaction protein; IL-6: } \\
\text { interleukin-6; PT: prothrombin time; APTT: activated partial thrombin time; ASA, American Society of } \\
\text { Anesthesiologists; TXA: Tranexamic acid. }\end{array}$} \\
\hline \multicolumn{4}{|c|}{$p$ value calculated using independent t-test, Pearson chi-square test or Fisher exact test. } \\
\hline *Significant difference & & & \\
\hline
\end{tabular}

Risk factors independently associated with opioid use were more operative time (OR [odds ratio] $=1.017$, $95 \% \mathrm{Cl}$ [confidence interval] $)=1.001$ to $1.032, p=0.034$ ). Moreover, the administration of TXA was a protective factor for opioid use after TKA (OR $=0.355,95 \% \mathrm{Cl}=0.161$ to $0.780, p=0.010)$ (Table 2). 
Table 2

Logistic regression models for opioid use following primary TKA

\begin{tabular}{|llll|}
\hline Parameter & Odds Ratio & $\mathbf{9 5 \%}$ Confidence Interval & $\boldsymbol{p}$ \\
\hline Smoking & 2.274 & $0.988-5.234$ & 0.054 \\
\hline TXA use & 0.355 & $0.161-0.780$ & $0.010^{*}$ \\
\hline Operative time & 1.017 & $1.001-1.032$ & $0.034^{\star}$ \\
\hline TXA: Tranexamic acid. & & \\
\hline *Significant difference & & \\
\hline
\end{tabular}

The LOS and related complication were summarized in Table 3. The LOS in opioid group was longer than that in non-opioid group ( $3.8 \pm 1.2$ vs $3.5 \pm 0.9, p=0.36)$. In addition, the incidence of nausea and vomiting was higher in opioid group $(25.3 \%$ vs $11.2 \%, p=0.001)$. The incidence of other complications, including deep venous thrombosis, pulmonary embolism, wound complications and so on, did not reach statistical difference between the two groups $(p>0.05)$

Table 3

LOS, expenses and Complications

\begin{tabular}{|c|c|c|c|}
\hline Variables & Opioids group, $\mathrm{N}=83$ & Non-opioids group, $\mathbf{N}=278$ & $p$ \\
\hline LOS & $3.8 \pm 1.2$ & $3.5 \pm 0.9$ & $0.036 *$ \\
\hline Transfusion & $1(1.2 \%)$ & $1(0.4 \%)$ & 1.000 \\
\hline Death & 0 & 0 & - \\
\hline DVT & 0 & $4(1.4 \%)$ & 0.489 \\
\hline $\mathrm{PE}$ & 0 & 0 & - \\
\hline Nausea and vomiting & $21(25.3 \%)$ & $31(11.2 \%)$ & $0.001^{*}$ \\
\hline Cardiac infarction & $1(1.2 \%)$ & 0 & 0.230 \\
\hline Stroke & 0 & 0 & - \\
\hline Acute renal failure & 0 & 0 & - \\
\hline Wound complications & $16(19.3 \%)$ & $37(13.3 \%)$ & 0.178 \\
\hline Readmission & $2(2.4 \%)$ & $2(0.7 \%)$ & 0.228 \\
\hline \multicolumn{4}{|c|}{$\begin{array}{l}\text { LOS: length of stay; DVT: deep venous thrombosis; PE: pulmonary embolism. Wound complications } \\
\text { included exudation, bleeding, swelling, infection and impaired wound healing. }\end{array}$} \\
\hline
\end{tabular}


Postoperative persistent pain following TKA is a collective concern for surgeons and patients [15]. Effective pain management after TKA could decrease complication risk, promote earlier rehabilitation, improve patient satisfaction and quality of life [16-18]. When conventional measures, such as NDAIDs and physical therapy, had poor efficacy, opioid drugs could be a remedy in this study. Thus, the risk factor associated with opioid use is the risk factors for poor pain control after primary TKA, actually. Because of the adverse effects of severe pain and opioid use, effective pain control and recognize the risk for opioid use could guide provider and hospital system approach to managing postoperative pain after TKA [18, 19].

Substantial previous studies focused on the prevalence and prolonged opioid use instead of simple opioid use after TKA $[9,11,12]$. A retrospective cohort study performed by Robert et al indicated that younger age, preoperative NSAIDs and opioid use and some comorbidities [12]. In addition, Bedard and his colleagues determined that preoperative opioid use, a higher comorbidity score, rheumatoid arthritis, smoking and benzodiazepine use were strong predictors for persistent opioid use following TKA [9]. The research conducted by Kim et al reached a similar conclusion [11]. To our best knowledge, this study is the first one to determine the risk factors for the postoperative opioid use following primary TKA. Following an enhanced-recovery programme, more operative time is a risk factor while TXA use is a protective risk for opioid use after primary TKA.

It was reported that operative time, intraoperative blood loss and postoperative pain in TKA were in close positive correlation. Extended operative time could lead to more intraoperative blood loss, further resulting in increasing pain after TKA $[20,21]$. Consequently, reducing operative time and blood loss was important steps for postoperative pain care. Besides, smoking might increase the risk for opioid use after TKA $(p=0.054)$. The probable reason was that smoking could cause more perioperative blood loss and higher complication risk [22]. Cryar et al demonstrated that preoperative smoking was the risk factor for narcotic use after TKA [23]. In addition, smoking were also a strong risk factor for persistent opioid use after primary TKA [11].

In this study, we found that application of TXA could reduce the risk of persistent pain and opioid use after TKA. As a antifibrinolytic agents, TXA could inhibit hyperfibrinolysis, reduce blood loss, alleviate joint swelling and relieve pain $[24,25]$. Related studies show that patients could gain less pain, less knee swelling and better knee function with the usage of multiple boluses of TXA following TKA $[25,26]$. In addition to reducing blood loss, TXA had a synergistic effect to relieve pain after orthopaedic procedures. Currently, the application of TXA is an essential measure in blood management in enhanced recovery protocols.

The patients in opioid group sustained worse pain, longer LOS, higher incidence of nausea and vomiting after primary TKA. Opioid use was associated with increased LOS after TKA, which was consistent with previous studies $[7,11]$. Halawi et al showed that opioid-based analgesia were significantly associated with increased LOS [7]. Another literature supported this result, showing that opioid use could lead to longer hospitalizations [10]. Moreover, nausea and vomiting, which could increase complication risk and 
delay postoperative recovery, may be the side effects of opioid use [27]. It was necessary to take measures to prevent nausea and vomiting.

There are some limitations in this study. Firstly, we do not enrolled preoperative medication history, such as sedative and opioid because related case data is incomplete. It was reported that preoperative benzodiazepine and opioid use were strong predictors for postoperative opioid use in TKA $[9,11]$. Secondly, as a retrospective study, all data is from electronic medical record system and the level of evidence is still low. In addition, the follow-up time is only 30 days while chronic pain after TKA may continue more than 6 months [11]. So the follow-up time in this study is insufficient to evaluate entire patients with opioid use after primary. In a word, further studies with higher evidence level are requisite.

\section{Conclusion}

Considering the adverse health effects of opioid use, strategies need to be developed to prevent persistent opioid use. Reducing operative time and application of tranexamic acid could lower the risk of opioid use with an enhanced-recovery programme after primary TKA.

\section{Declarations}

Compliance with ethical standards

Conflict of interest All authors declare no conflict of interest.

Informed consent Informed consent was obtained from all individual participants included in the study.

\section{References}

1. Kirksey M, Chiu YL, Ma Y, Della Valle AG, Poultsides L, Gerner P, Memtsoudis SG: Trends in in-hospital major morbidity and mortality after total joint arthroplasty: United States 1998-2008. Anesthesia and analgesia 2012, 115(2):321-327.

2. Steele T, Eidem L, Bond J: Impact of Adoption of Smart Pump System With Continuous Capnography Monitoring on Opioid-Related Adverse Event Rates: Experience From a Tertiary Care Hospital. Journal of patient safety 2019.

3. Lei YT, Xu B, Xie XW, Xie JW, Huang Q, Pei FX: The efficacy and safety of two low-dose peri-operative dexamethasone on pain and recovery following total hip arthroplasty: a randomized controlled trial. International orthopaedics 2018, 42(3):499-505.

4. Xie X, Pei F, Huang Z, Tan Z, Yang Z, Kang P: Does patellar denervation reduce post-operative anterior knee pain after total knee arthroplasty? Knee surgery, sports traumatology, arthroscopy : official journal of the ESSKA 2015, 23(6):1808-1815.

5. Hochberg MC, Altman RD, April KT, Benkhalti M, Guyatt G, McGowan J, Towheed T, Welch V, Wells G, Tugwell P: American College of Rheumatology 2012 recommendations for the use of 
nonpharmacologic and pharmacologic therapies in osteoarthritis of the hand, hip, and knee. Arthritis care \& research 2012, 64(4):465-474.

6. da Costa BR, Nüesch E, Kasteler R, Husni E, Welch V, Rutjes AW, Jüni P: Oral or transdermal opioids for osteoarthritis of the knee or hip. The Cochrane database of systematic reviews 2014(9):Cd003115.

7. Halawi MJ, Vovos TJ, Green CL, Wellman SS, Attarian DE, Bolognesi MP: Opioid-Based Analgesia: Impact on Total Joint Arthroplasty. The Journal of arthroplasty 2015, 30(12):2360-2363.

8. Inacio MC, Pratt NL, Roughead EE, Paxton EW, Graves SE: Opioid use after total hip arthroplasty surgery is associated with revision surgery. BMC musculoskeletal disorders 2016, 17:122.

9. Bedard NA, Pugely AJ, Westermann RW, Duchman KR, Glass NA, Callaghan JJ: Opioid Use After Total Knee Arthroplasty: Trends and Risk Factors for Prolonged Use. The Journal of arthroplasty 2017, 32(8):2390-2394.

10. Jones MR, Kramer ME, Beutler SS, Kaye AD, Rao N, Brovman EY, Urman RD: The Association Between Potential Opioid-Related Adverse Drug Events and Outcomes in Total Knee Arthroplasty: A Retrospective Study. Advances in therapy 2020, 37(1):200-212.

11. Kim SC, Choudhry N, Franklin JM, Bykov K, Eikermann M, Lii J, Fischer MA, Bateman BT: Patterns and predictors of persistent opioid use following hip or knee arthroplasty. Osteoarthritis and cartilage 2017, 25(9):1399-1406.

12. Namba RS, Singh A, Paxton EW, Inacio MCS: Patient Factors Associated With Prolonged Postoperative Opioid Use After Total Knee Arthroplasty. The Journal of arthroplasty 2018, 33(8):2449-2454.

13. Ripolles-Melchor J, Abad-Motos A, Diez-Remesal Y, Aseguinolaza-Pagola M, Padin-Barreiro L, Sanchez-Martin R, Logrono-Egea M, Catala-Bauset JC, Garcia-Orallo S, Bisbe E et al: Association Between Use of Enhanced Recovery After Surgery Protocol and Postoperative Complications in Total Hip and Knee Arthroplasty in the Postoperative Outcomes Within Enhanced Recovery After Surgery Protocol in Elective Total Hip and Knee Arthroplasty Study (POWER2). JAMA surgery 2020:e196024.

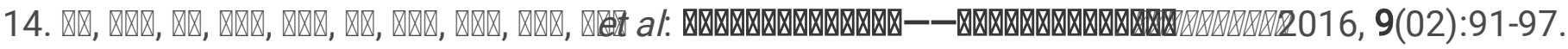

15. Nilsdotter AK, Toksvig-Larsen S, Roos EM: Knee arthroplasty: are patients' expectations fulfilled? A prospective study of pain and function in 102 patients with 5-year follow-up. Acta orthopaedica 2009, 80(1):55-61.

16. Elmallah RK, Chughtai M, Khlopas A, Newman JM, Stearns KL, Roche M, Kelly MA, Harwin SF, Mont MA: Pain Control in Total Knee Arthroplasty. The journal of knee surgery 2018, 31(6):504-513.

17. Gonzales J, Lovald ST, Lau EC, Ong KL: Risk of Opioid-Related Adverse Events After Primary and Revision Total Knee Arthroplasty. Journal of surgical orthopaedic advances 2018, 27(2):148-154.

18. Shafi S, Collinsworth AW, Copeland LA, Ogola GO, Qiu T, Kouznetsova M, Liao IC, Mears N, Pham AT, Wan GJ et al: Association of Opioid-Related Adverse Drug Events With Clinical and Cost Outcomes Among Surgical Patients in a Large Integrated Health Care Delivery System. JAMA surgery 2018, 153(8):757-763. 
19. Baratta JL, Gandhi K, Viscusi ER: Perioperative pain management for total knee arthroplasty. Journal of surgical orthopaedic advances 2014, 23(1):22-36.

20. Friedman RJ: Limit the bleeding, limit the pain in total hip and knee arthroplasty. Orthopedics 2010, 33(9 Suppl):11-13.

21. Hrnack SA, Skeen N, Xu T, Rosenstein AD: Correlation of body mass index and blood loss during total knee and total hip arthroplasty. American journal of orthopedics (Belle Mead, NJ) 2012, 41(10):467471.

22. Manchikanti L, Singh A: Therapeutic opioids: a ten-year perspective on the complexities and complications of the escalating use, abuse, and nonmedical use of opioids. Pain physician 2008, 11(2 Suppl):S63-88.

23. Cryar KA, Hereford T, Edwards PK, Siegel E, Barnes CL, Mears SC: Preoperative Smoking and Narcotic, Benzodiazepine, and Tramadol Use are Risk Factors for Narcotic Use After Hip and Knee Arthroplasty. The Journal of arthroplasty 2018, 33(9):2774-2779.

24. Hogan CA, Golightly LK, Phong S, Dayton MR, Lyda C, Barber GR: Perioperative blood loss in total hip and knee arthroplasty: Outcomes associated with intravenous tranexamic acid use in an academic medical center. SAGE open medicine 2016, 4:2050312116637024.

25. Xie J, Ma J, Yao H, Yue C, Pei F: Multiple Boluses of Intravenous Tranexamic Acid to Reduce Hidden Blood Loss After Primary Total Knee Arthroplasty Without Tourniquet: A Randomized Clinical Trial. The Journal of arthroplasty 2016, 31(11):2458-2464.

26. Lei Y, Huang Q, Huang Z, Xie J, Chen G, Pei F: Multiple-Dose Intravenous Tranexamic Acid Further Reduces Hidden Blood Loss After Total Hip Arthroplasty: A Randomized Controlled Trial. The Journal of arthroplasty 2018, 33(9):2940-2945.

27. Ryu JH, Jeon YT, Min B, Hwang JY, Sohn HM: Effects of palonosetron for prophylaxis of postoperative nausea and vomiting in high-risk patients undergoing total knee arthroplasty: $A$ prospective, randomized, double-blind, placebo-controlled study. PloS one 2018, 13(5):e0196388. 